\title{
Masculinidad y feminidad en hombres jóvenes mexicanos. ¿Un asunto de orientación sexual?
}

\section{Masculinity and Feminity in Mexican Young Men: A Matter of Sexual Orientation?}

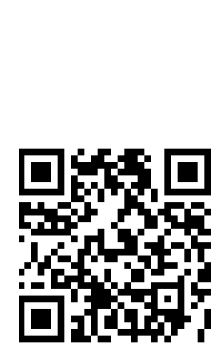

\author{
Jorge García-Villanueva ${ }^{1}$ \\ Universidad Pedagógica Nacional \\ Ciudad de México \\ jvillanueva@upn.mx \\ Denize Maday Meza-Mercado² \\ Instituto Nacional de Psiquiatría \\ Ciudad de México \\ denize@imp.mx \\ Claudia Ivonne Hernández-Ramírez ${ }^{3}$ \\ Secretaría de Educación Pública \\ Ciudad de México \\ civonneramirez@hotmail.com \\ Daniel Moreno-García ${ }^{4}$ \\ Secretaría de Educación Pública \\ Ciudad de México \\ daniel.moreno@sep.gob.mx
}

Recibido 1 de marzo de 2016 • Corregido 7 de abril de 2017• Aceptado 25 de abril de 2017

\begin{abstract}
${ }^{1}$ Doctor en Psicología por la Facultad de Psicología de la Universidad Nacional Autónoma de México (UNAM), profesor titular definitivo en la Universidad Pedagógica Nacional (UPN), Ajusco. Es especialista en estudios de género en tópicos relativos a juventud, identidad, masculinidad, violencia y formación profesional. Es responsable de la Especialidad de Género en Educación, de la UPN. Cuenta con decenas de tesis dirigidas en licenciatura y en posgrado. Es coautor de dos libros sobre valores y género en educación y uno más sobre identidad masculina, también de varios artículos indexados, capítulos de libro, artículos de divulgación y reseñas. Ha participado como ponente, instructor, conferencista y
\end{abstract} evaluador en diversas instituciones.

${ }^{2}$ Psicóloga por la Facultad de Psicología de la Universidad Nacional Autónoma de México (UNAM) y cuenta con estudios de maestría en bioética por su alma mater. Actualmente labora en el Instituto Nacional de Psiquiatría "Ramón de la Fuente Muñiz" como investigadora asociada en el área de Investigaciones Epidemiológicas y Psicosociales.

${ }^{3}$ Licenciada en Psicología Educativa y Especialista de Género en Educación por la Universidad Pedagógica Nacional (UPN), Ajusco. Actualmente, se desempeña como maestra, psicóloga y orientadora para Educación Básica y Educación Especial, en la Secretaría de Educación Pública (SEP). Ha sido asesora psicopedagógica en programas de desarrollo de competencias en alumnado de bajo rendimiento escolar y alfabetización académica, además, ha brindado tutoría a tesistas de nivel licenciatura. También ha colaborado en campañas de asesoramiento, acompañamiento y apoyo a personas en situaciones de riesgo. Partícipe en coloquios, exposiciones y conferencias vinculadas al ámbito educativo.

${ }^{4}$ Licenciado en Psicología Educativa por la Universidad Pedagógica Nacional (UPN), Ajusco donde es asistente de investigación desde 2013. Actualmente colabora para la Secretaría de Educación Pública (SEP). 
doi: http://dx.doi.org/10.15359/ree.21-2.16

URL: http://www.una.ac.cr/educare

CORREO: educare@una.cr

Resumen: La investigación de la masculinidad y feminidad en función de la orientación sexual es un asunto escasamente analizado en la Ciudad de México. En este estudio fueron comparados los resultados obtenidos de la aplicación del Inventario de Masculinidad y Feminidad (IMAFE) a dos muestras de varones: una homosexual y otra heterosexual, ambas con edades entre los 17 y los 25 años. La primera, constó de 56 y la segunda de 57 sujetos, para un total de 113 personas. La selección de los participantes fue por medio de un muestreo no probabilístico arbitrario intencional. En su mayoría, participaron estudiantes de licenciatura y un porcentaje mínimo de bachillerato. El tipo de estudio fue descriptivo y el diseño no experimental, se observó el fenómeno en su contexto. Después de calificar cada una de las pruebas se procedió a analizar los datos mediante la prueba de comparación $t$ de Student. Los resultados obtenidos mostraron que no hubo diferencias estadísticamente significativas $(p<0.50)$ en las escalas del IMAFE. Se concluye que no hay diferencias en la masculinidad y feminidad de los sujetos en función de su orientación sexual, aunque se observa una tendencia a la androginia. Los hallazgos de esta exploración podrán utilizarse como aspectos que contribuyan a una educación que favorezca la tolerancia a la diversidad y, por ende, el desmantelamiento de obcecaciones en torno a las personas homosexuales.

Palabras claves: Género; masculinidad; feminidad; homosexualidad y heterosexualidad.

\begin{abstract}
The relationship between masculinity and femininity in terms of sexual orientation is scarcely analyzed in Mexico City. This study compared the results obtained from the application of the Masculinity and Femininity Inventory (IMAFE) to two samples of men: one homosexual, and one heterosexual; both groups aged between 17 and 25 years. The first sample consisted of 56 subjects, and the second one was of 57, for a total of 113 people. Most of them had a degree, and a minimum percentage of the participants had an associate's degree. After grading the tests, the data were analyzed by using the Student's t-test. The results showed that there were no statistically significant differences in the IMAFE scales. It is concluded that there are no differences in the masculinity and femininity of the subjects according to their sexual orientation, although a tendency to androgyny is observed. The findings of this exploration can be used as aspects contributing to an education that favors the tolerance to the diversity and, therefore, the dismantling of obscenities toward the homosexual people.
\end{abstract}

Keywords: Gender; masculinity; feminity; homosexuality; heterosexuality.

Dear Mrs.

I gather from your letter that your son is a homosexual. I am most impressed by the fact, that you do not mention this term yourself in your information about him. May I question you why you avoid it? Homosexuality is assuredly no advantage, but it is nothing to be ashamed of, no vice, no degradation, it cannot be classified as an illness; we consider it to be a variation of the sexual function produced by a certain arrest of sexual development. Many highly respectable individuals of ancient and modern times have been homosexuals, several of the greatest men among them (Plato, Michelangelo, Leonardo da Vinci, etc.) It is a great injustice to persecute homosexuality as a crime and cruelty too. If you do not believe me, read the books of Havelock Ellis. 
[Estimada señora:

Por su carta entiendo que su hijo es homosexual. Me impresiona el hecho de que usted misma no mencione esta palabra al informarme sobre él. ¿Puedo preguntarle por qué la evita? La homosexualidad no es sin duda una ventaja, pero tampoco algo de lo que avergonzarse, no es un vicio, no es una degradación, y no puede catalogarse como una enfermedad; lo consideramos una variación de la función sexual producida por una cierta detención en el desarrollo. Muchas personas respetables de los tiempos antiguos y modernos han sido homosexuales, entre ellos, algunos grandes hombres (Platón, Miguel Ángel, Leonardo Da Vinci, etcétera). Es una gran injusticia y una crueldad perseguir la homosexualidad como si fuera un delito. Si no me cree, lea los libros de Havelock Ellis]. (Historical Notes. A letter from Freud, 1951)

\section{Introducción}

La percepción de hombres y mujeres ha estado basada en una visión bipolar de corte biologicista, fundamentada en las funciones reproductoras, según el modelo de la familia nuclear. Desde 1960, la tendencia del estudio de los papeles de género se ha abordado desde el enfoque llamado construcción social del género. Se sabe que las creencias y percepciones de las habilidades y actividades diferenciales de varones y mujeres pueden afectar al comportamiento, confirmando las creencias genéricas que las personas tienen sobre sí mismas.

Esto significa, como advierte Fernández (1998), que en la medida en que el sexo sigue siendo un factor organizador central de las relaciones sociales, no es posible saber hasta qué punto el comportamiento diferencial pueda ser debido a causas internas, sean atribuidas a la biología, a la socialización temprana o a la personalidad diferencial de los sexos. A través de la presente investigación de la masculinidad-feminidad en función de la preferencia sexual, se pretende dar un acercamiento científico al estudio de este fenómeno, con la finalidad de evaluar algunos prejuicios en torno a los hombres homosexuales, prejuicios que, en general, señalan que los hombres con esta preferencia son muy femeninos y poco masculinos (Fernández, 1998).

\section{¿Es lo mismo sexo y género? \\ Un acercamiento a la construcción de la identidad masculina y femenina}

Los seres humanos sufren una diferenciación/sexuación, a lo largo de los nueve meses de gestación, dicha diferenciación se da a nivel genético, endocrinológico, anatomofisiológico y neurológico; lo cual da como resultado la forma visible en hombre o mujer (Fernández, 1998). Se entiende por sexo la condición orgánica que distingue al macho de la hembra y por extensión se emplea para designar los órganos característicos de cada sexo, órganos sexuales internos y externos. 
doi: http://dx.doi.org/10.15359/ree.21-2.16

URL: http://www.una.ac.cr/educare

CORREO: educare@una.cr

Una vez que ha nacido el nuevo ser las personas que lo rodean se comportarán de acuerdo con el sexo que presente, le asignarán un nombre, lo vestirán de rosa o azul, le dirán a qué y cómo jugar, etcétera. Este comportamiento dará lugar a un aprendizaje por parte del neonato, mismo que adoptará como su deber ser, a lo cual se denomina género.

Para diferenciar sexo y género se considera que el sexo se refiere al hecho biológico de la diferenciación sexual de los seres humanos, en tanto que el género tiene relación con los significados que cada grupo social le atribuye a tal hecho. Así, los sistemas de género, según De Barbieri (1993), "son los conjuntos de prácticas, símbolos, representaciones, normas y valores sociales que las sociedades elaboran a partir de la diferencia sexual anatomo-fisiológica y que dan sentido a la satisfacción de los impulsos sexuales, a la reproducción de las [sic] especie humana y en general [a las relaciones] entre las personas" (p. 149-150).

Respecto a la adquisición del género, en los primeros años de vida, se empieza a desarrollar una imagen corporal que permite que las niñas y los niños puedan distinguir las características del cuerpo; entonces se identifican con algunas de las imágenes femeninas o masculinas y se categorizan dentro de uno de los dos géneros (Heilman, 2012). Es así como el proceso de adopción de los roles de género tiene dos facetas en las que está el descubrimiento de estos mismos y la discriminación en la adopción de estos, según se trate de un varón o de una hembra. A partir de ello, madres y padres aplican el reforzamiento diferencial, comportándose de manera distinta con niños que con niñas, y es desde este momento que interiorizan aquellas cualidades que la sociedad espera (Martin, Ruble y Szkrybalo, 2002).

Cada cultura, al construir el marco de referencia de las personas, parte de una serie de premisas histórico-socio-culturales entendidas como las tradiciones respecto a los valores, creencias, pensamientos y acciones (Lippa, 2010), lo cual puede llevar a pensar que la adquisición y desarrollo del género es un proceso multifactorial. En este sentido, es necesario comprender que el sexo y el género son culturalmente construidos e históricamente situados, las categorías dicotómicas de 'femenino', 'masculino', 'heterosexual' y 'homosexual' según Butler, Laclau y Žižek (2003), son la repetición de actos performativos en lugar de valores naturales o innatos. El género y el sexo son resultado de actuaciones, de actos performativos derivados del discurso autoritario creador del poder hegemónico centrado en la heteronormatividad obligatoria (Duque, 2010).

\section{¿Y, ahora qué, decido ser heterosexual u homosexual?}

En la mayoría de las sociedades la heterosexualidad es vista como la normalidad, en términos de relaciones sexuales y sociales, y a su vez, la homosexualidad tiende a ser calificada como anormal. No obstante, es probable que la homosexualidad exista desde los orígenes de la humanidad. En la actualidad, hay sociedades que aceptan y toleran algunas formas de homosexualidad. En este sentido, Giménez (1992) menciona que el límite entre la sexualidad 
normal y anormal se encuentra establecido por los criterios subjetivos del individuo y, sobre todo, por los límites que imponen la cultura y la sociedad.

El término homosexualidad ha tenido una variedad de cambios conceptualmente, de acuerdo con Garrabe (1993), esta "quimera grecolatina" se forjó sobre el modelo de numerosas palabras técnicas cuyo primer elemento es homos (omos), semejante; para designar el atractivo erótico por personas del mismo sexo.

En la primera mitad del siglo XIX, la homosexualidad fue considerada como una inversión congénita del sentimiento sexual, es decir, contrario sexual. De acuerdo con González (2001), la homosexualidad se consideró como una de las formas de manifestación de tal inversión. Al respecto, Álvarez y Mazin (1990), recuperando el planteamiento de la obra "Psycopathia Sexualis" de Von Krafft-Ebing (2012), plantean que la homosexualidad era vista como una parestesia porque existía una ausencia de sentimiento genésico (carnal) hacia el otro sexo, en tanto que había tendencia e instinto hacia el propio.

Según Dorsch (1976, citado en Olvera, 1997), la homosexualidad u homoerotismo es el amor sexual entre personas del mismo sexo, inversión sexual. Para Bastin (1979) la homosexualidad del sujeto soltero y la del sujeto adolescente tienen más facilidades de entrar en relaciones con personas de su mismo sexo, son a menudo accidentales; pero, por efecto de las circunstancias y de la inmadurez de carácter, pueden transformarse en una homosexualidad esencial. En el caso adolescente, cabría hablar de homosexualidad evolutiva, etapa posible y no necesaria para pasar del narcisismo a la relación heterosexual.

Lo que la persona homosexual persigue en un compañero o compañera del mismo sexo es otro yo, un espejo que le devuelva su propia imagen y que, en el caso del varón, le tranquilice en lo que respecta a su propia virilidad. Tal es la razón de que las relaciones homosexuales sean tan frecuentes en la adolescencia (Rubio y Aldana, 1994). La manifestación de una conducta homosexual puede presentarse cuando se está restringido el acceso a parejas heterosexuales; tal es el caso de las prisiones, seminarios, guerras, etcétera. En 1981, Money, consideró que los procesos de vinculación son el elemento central de la homosexualidad; formar un par eróticosexual con un compañero que presenta una morfología genital idéntica y que en ella incluye al enamoramiento en la manifestación compleja. La expresión homosexualidad indica la unión de dos personas del mismo sexo (Valentova, Rieger, Havlicek, Linsenmeier y Bailey, 2011).

En las publicaciones especializadas se encuentra también la palabra inversión, que indica la transformación de los sentimientos en dirección contraria a la habitual; y lo mismo se dice del concepto de sexualidad contraria. Una definición más ha sido la que Álvarez y Mazin (1990) elaboraron, al considerar que la homosexualidad es el gusto o la preferencia para relacionarse afectiva o eróticamente con personas del mismo sexo. 
doi: http://dx.doi.org/10.15359/ree.21-2.16

URL: http://www.una.ac.cr/educare

CORREO: educare@una.cr

En resumen, la homosexualidad constituye una actividad erótico-sexual en la que participan miembros de un mismo sexo; el contacto en este caso, por lo general, conduce al orgasmo. Algunos sexólogos han considerado, desde la década de los setenta, que la experiencia homosexual es tan diversa, y los aspectos psicológicos, sociales y sexuales relacionados con ella tan variados, que el uso de las palabras "homosexual" u "homosexualidad" para describir solo la elección como pareja de un individuo del mismo sexo en un momento particular es confuso e inexacto (McCary, McCary, Álvarez-Gayou, Del Río y Suáez, 1996).

El elemento que, al parecer, define la preferencia es el de la atracción, y no solamente la sexual y erótica, sino también el hecho simple y cotidiano de que las personas enfoquen su atención visual, sobre todo en personas de un sexo: las mujeres o los hombres. La atracción se experimenta hacia miembros de un género en particular, es decir, hacia las cualidades fenotípicas y externas; lo que caracteriza a una persona como hombre o mujer. En este sentido, es preferible el uso del término preferencia genérica en sustitución de orientación sexual (McCary et al., 1996).

Sinembargo,tantola sexualidad canónica, hegemónica,comolatransgresora,"ininteligible", se construyen mediante la performatividad, es decir, por medio de la repetición ritualizada (iteración) de actos de habla y de todo un repertorio de gestos corporales que obedecen a un estilo relacionado con uno de los dos géneros culturales. Esta repetición ritualizada, en palabras de Butler (2002) no es opcional, sino que se basa en un discurso regulativo, una exigencia constante del entorno, encaminada a producir aquellos fenómenos que regulan y constriñen la conducta en relación con la identidad sexual.

Desde lo que postula Butler et al. (2003) se considera que el sujeto heterosexual como el sujeto perteneciente a la amplia gama que corresponde a la diversidad sexual, son el efecto y resultado de la producción de una red de dispositivos de saber/poder que se explicitan en las concepciones esencialistas, imperantes actualmente, del género y la diferencia sexual.

Desde esta lógica, la palabra homosexualidad tiene diferentes significados y las definiciones varían según la época, el contexto sociohistórico y su cariz se matiza conforme al criterio arbitrariamente sentado por personas que desean recalcar diversos aspectos de la homosexualidad (Álvarez y Mazin, 1990), por consiguiente, la conducta homosexual puede aparecer en una gama muy amplia de circunstancias. La conceptualización de la homosexualidad debe hacerse a partir de la experiencia interna de la persona, la cual puede considerarse una preferencia erótica, afectiva o sexual hacia gente del mismo sexo, relativamente continua, que incide en el estilo de vida de quien la ejerce, sin que ello sea considerado una psicopatología. 


\section{Método}

El tipo de estudio de esta investigación fue descriptivo porque se buscó decir cómo es el fenómeno orientación sexual, masculinidad y feminidad (Hernández, Fernández-Collado y Baptista, 2000). De corte transversal, debido a que se recolectaron los datos en un solo momento y en un tiempo único; y comparativo porque se utilizaron dos grupos para confrontar sus resultados. El diseño es no experimental, puesto que no se manipularon intencionalmente las variables independientes, es decir, se observó el fenómeno en su contexto.

\section{Participantes}

Los sujetos, en su totalidad, fueron hombres de entre 17 a 25 años, estudiantes de licenciatura y una minoría de bachillerato. Las pruebas fueron aplicadas en distintos lugares de la Ciudad de México (alrededores de Ciudad Universitaria, Polanco, la Zona Rosa, Chapultepec, y de la delegación Iztacalco). La selección de las personas participantes se hizo por muestreo no probabilístico arbitrario intencional (Hernández et al., 2000). Se aplicó un total de 150 pruebas y se procedió a descartar a los sujetos que rebasaran la edad de 25 años o fueran menores de 17 años, de lo cual se conformaron los grupos: 56 personas en el homosexual y 57 personas en el heterosexual.

El instrumento empleado fue el Inventario de masculinidad y feminidad (IMAFE) elaborado por Lara (1993), la finalidad del diseño fue para que midiera papeles de género de manera confiable y válida en México. El IMAFE es un inventario que mide masculinidad, feminidad, machismo y sumisión, además está basado en los aspectos más representativos de los roles y estereotipos en la cultura mexicana y, a la vez, incluye algunos explorados en otros países, donde se le pide a la persona que responda a 60 reactivos con una escala de siete números (del 1 al 7); cada uno de los ítems corresponde a una escala diferente de los tres reactivos anteriores a ese y los tres reactivos posteriores (ver Apéndice A).

Una vez localizados algunos puntos de reunión de personas homosexuales se procedió a la aplicación del IMAFE. Se le explicó a cada participante el motivo de la investigación, el carácter confidencial de los datos y se acordó un consentimiento informado de su colaboración en un estudio psicológico, en el cual se le garantizaría anonimato y resguardo en sus respuestas. Asimismo, se le precisó que en la hoja de datos personales del IMAFE indicara lo que se le pedía, y si deseaba información sobre su cuestionario podía incluir la dirección de su correo electrónico.

\section{Resultados}

Después de haber aplicado y calificado todas las pruebas se procedió a realizar la base de datos correspondiente en el programa estadístico SPSS. Fueron capturadas las calificaciones de cada escala obtenidas por cada sujeto, así como la variable nominal edad. 
doi: http://dx.doi.org/10.15359/ree.21-2.16

URL: http://www.una.ac.cr/educare

CORREO: educare@una.cr

Para conocer la edad de ambos grupos, se realizó un análisis de frecuencias de dicha variable. De este análisis se obtuvo que en el grupo homo la edad con mayor frecuencia fue de 22 años mientras que para el grupo hetero la edad con mayor frecuencia fue de 21 años; cabe mencionar, que las edades del grupo hetero se distribuyeron de manera uniforme a diferencia de las del grupo homo. La media para ambos grupos fue 21 años, como se muestra en la Tabla 1.

Tabla 1: Medidas de tendencia central para las edades de los grupos homo y hetero

\begin{tabular}{ccc}
\hline Medida & Grupo homo & Grupo hetero \\
\hline Media & 20.94 & 21.26 \\
\hline
\end{tabular}

Del mismo modo, se procedió a observar la frecuencia de la escolaridad de los grupos; se distingue que la mayor parte de los sujetos cuenta con escolaridad de nivel licenciatura (Tabla 2).

Tabla 2: Distribución de la escolaridad por grupo

\begin{tabular}{ccc}
\hline Grupo & Bachillerato & Licenciatura \\
\hline Homo $(n=56)$ & 15 & 41 \\
Hetero $(n=57)$ & 4 & 57 \\
\hline
\end{tabular}

De la comparación de las medias obtenidas por Lara (1993) en la estandarización del IMAFE con las resultantes de esta investigación, se observa que la masculinidad del grupo homo tiene un promedio mayor que las medias del grupo hetero y del IMAFE; lo mismo sucede con la escala de feminidad. Como se distingue en la Tabla 3, el grupo hetero tiene menos discrepancia con las medias del IMAFE, e incluso entre sus escalas M y F.

De lo anterior, puede decirse que el grupo homo es tanto más femenino como más masculino que el heterosexual; amén de que sus escalas tienden a medir lo mismo, a lo cual Lara (1993) define como androginia.

Tabla 3: Comparación, por escala entre el IMAFE y los grupos homo y hetero

\begin{tabular}{cccc}
\hline Escala & $\begin{array}{c}\text { IMAFE } \\
\text { (media y desv. estándar) }\end{array}$ & $\begin{array}{c}\text { Grupo hetero IMAFE (media } \\
\text { y desv. estándar) }\end{array}$ & $\begin{array}{c}\text { Grupo homo IMAFE (media } \\
\text { y desv. estándar) }\end{array}$ \\
\hline Masculinidad & $4.76(0.87)$ & $4.84(0.88)$ & $4.90(0.63)$ \\
Feminidad & $4.34(1.14)$ & $4.48(1.04)$ & $4.83(1.01)$ \\
\hline
\end{tabular}


Como resultado de la aplicación de la prueba t de Student se obtuvo que las desviaciones estándar (ver tabla 4) para ambas escalas en el grupo homo son más cercanas a las del IMAFE en comparación con las del grupo hetero; lo que significa que el grupo homosexual discrepa menos o se desvía menos de la media establecida por el IMAFE. En otras palabras, la población del grupo homosexual se parece más a la del IMAFE (ver Tabla 4).

Tabla 4: Comparación, por escala, de las desviaciones estándar entre el IMAFE y los grupos homo y hetero

\begin{tabular}{cccc}
\hline Escala & IMAFE & Grupo hetero & Grupo homo \\
\hline Masculinidad & 0.87 & 0.88 & 0.63 \\
Feminidad & 1.14 & 1.04 & 1.01 \\
\hline
\end{tabular}

Asimismo, la prueba t de Student (Tabla 5) mostró que la escala de masculinidad no varía mucho entre ambos grupos; donde se encuentran diferencias importantes es en la feminidad, pues la del grupo homo es mucho más alta que la del hetero.

Tabla 5. Resultados de la prueba t de Student arrojados por el programa SPSS para Windows

\begin{tabular}{|c|c|c|c|c|c|}
\hline \multicolumn{6}{|c|}{ Prueba T de Student } \\
\hline & & \multicolumn{2}{|c|}{ Diferencias pareadas } & \multirow[t]{2}{*}{$\mathrm{T}$} & \multirow[t]{2}{*}{ Significancic } \\
\hline & & Media & Desviación estándar & & \\
\hline Par 1. & Masculinidad homo vs. hetero & 0.04 & \pm 0.96 & 0.37 & 0.70 \\
\hline Par 2. & Feminidad homo vs. hetero & 0.37 & \pm 1.40 & 1.97 & 0.05 \\
\hline
\end{tabular}

Nota: Nivel se significancia $p(p<0.50)$.

En términos generales, se puede decir que la masculinidad del grupo homo (ver Figura 1) concuerda con los parámetros del IMAFE y con las medidas del grupo hetero (ver Figuras 2, 3 y 4); pero su feminidad es mucho más alta en comparación con ambos. 
doi: http://dx.doi.org/10.15359/ree.21-2.16

URL: http://www.una.ac.cr/educare

CORREO: educare@una.cr

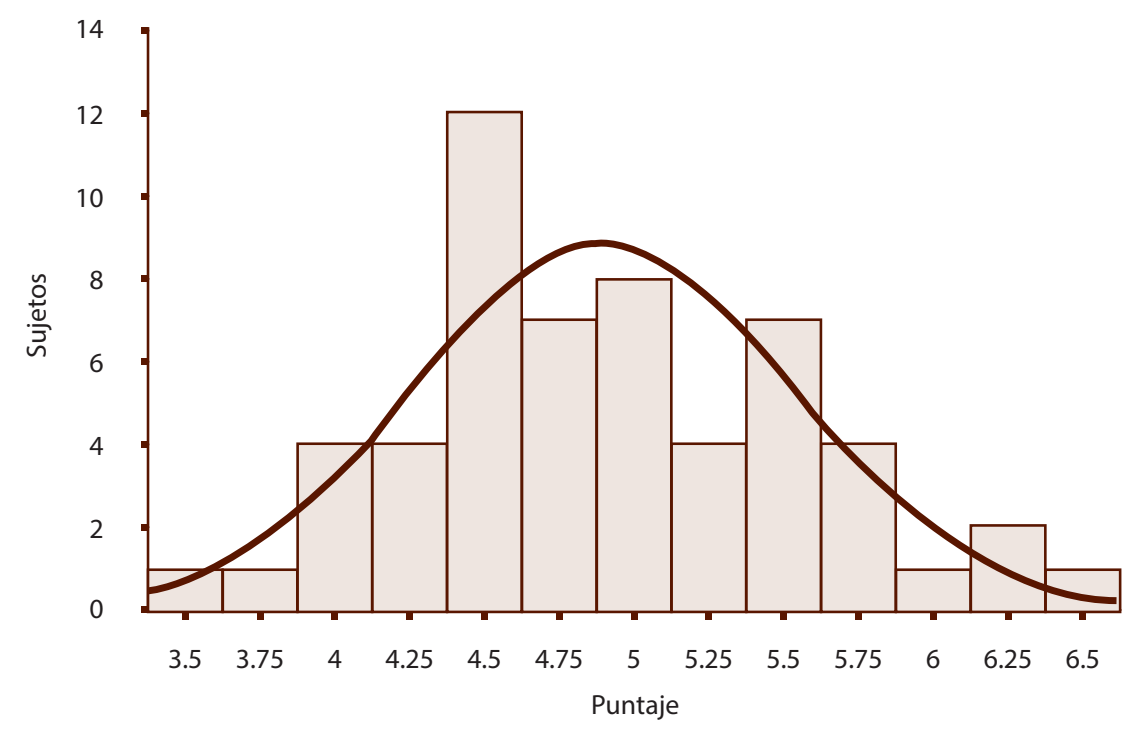

Figura 1: Distribución de los puntajes de masculinidad del grupo homo.

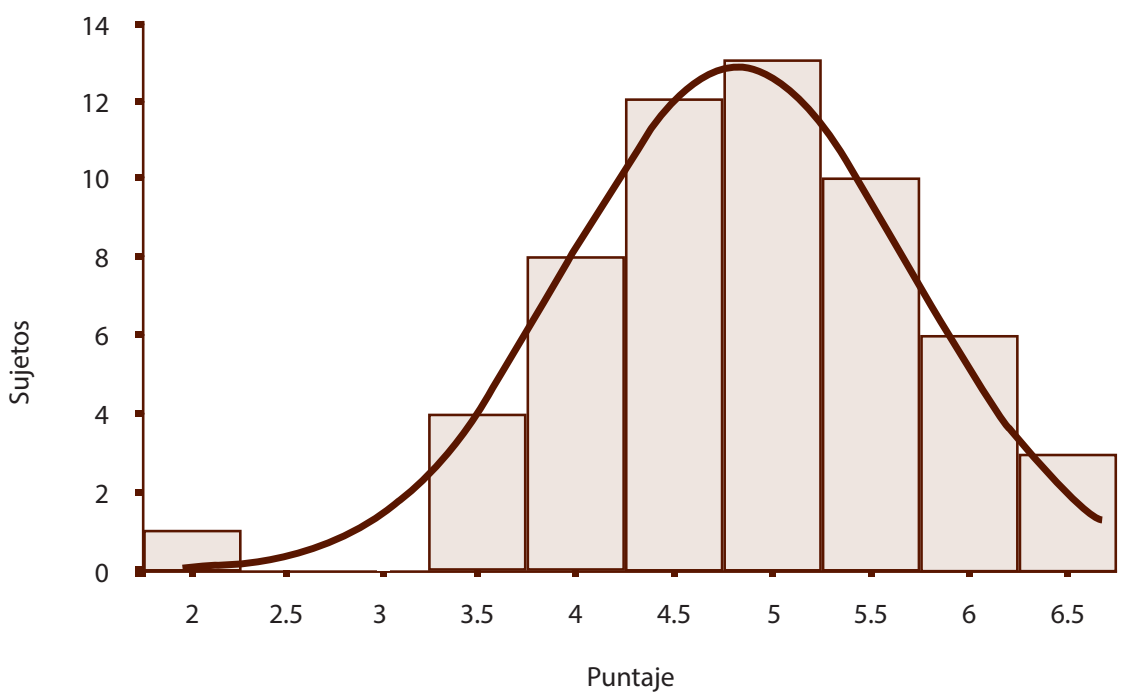

Figura 2: Distribución de los puntajes de masculinidad del grupo del grupo hetero. 
doi: http://dx.doi.org/10.15359/ree.21-2.16

URL: http://www.una.ac.cr/educare

CORREO: educare@una.cr

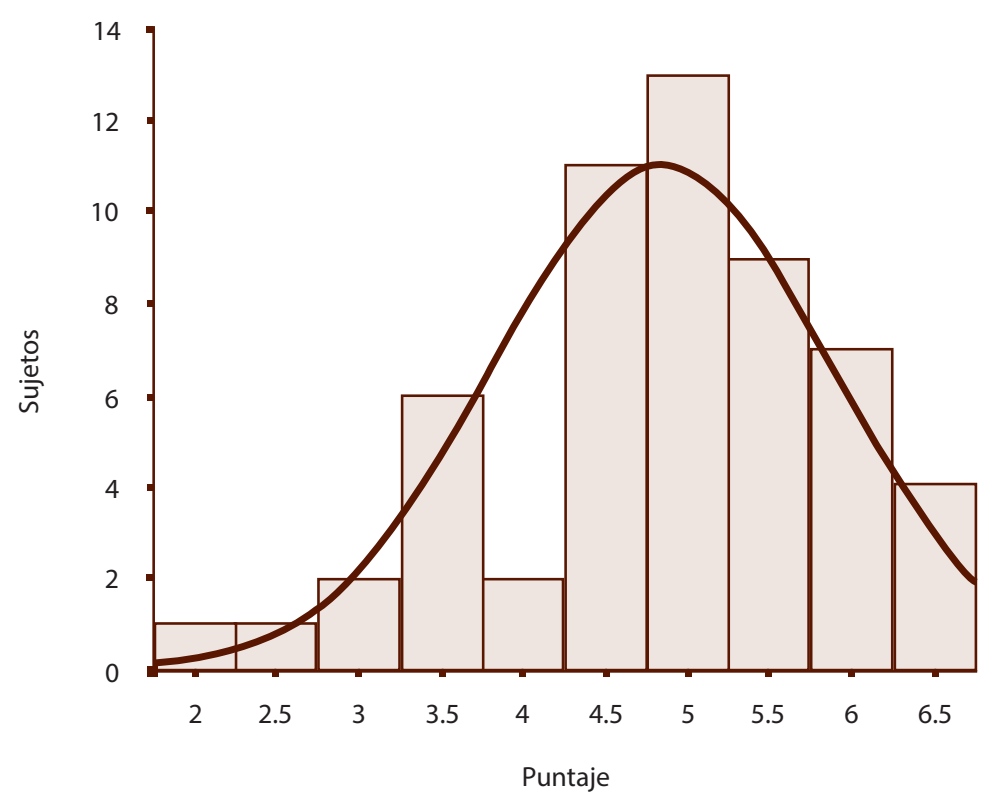

Figura 3: Distribución de los puntajes de feminidad del grupo homo.

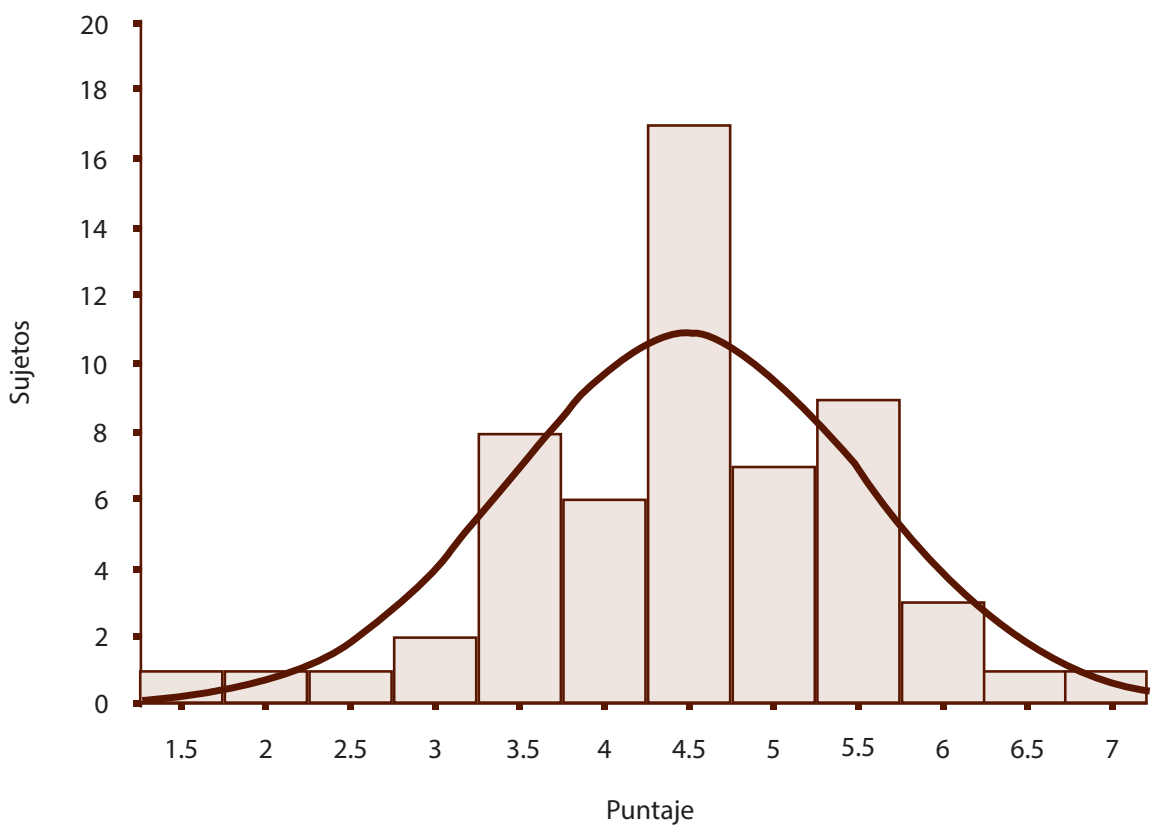

Figura 4: Distribución de los puntajes de feminidad del grupo hetero. 
doi: http://dx.doi.org/10.15359/ree.21-2.16

URL: http://www.una.ac.cr/educare

CORREO: educare@una.cr

\section{Discusión}

La investigación de la masculinidad y feminidad ha sido poco estudiada, en función de la orientación sexual. La realización de este trabajo permitió el acercamiento científico al estudio de dicho fenómeno, con la finalidad de evaluar algunos prejuicios en torno a las personas homosexuales que, en general, señalan que los hombres con esta preferencia son muy femeninos y poco masculinos.

De acuerdo con los resultados derivados del análisis estadístico, se puede decir que no existen diferencias estadísticamente significativas $(p<0.50)$ entre los rasgos M y F del IMAFE al comparar los grupos homosexual y heterosexual, pues el conjunto de los datos se encuentra aún dentro de los parámetros considerados por el mismo instrumento (ver Tablas 3 y 4).

Cabe señalar que el hecho de que las medias obtenidas en este estudio fueran mayores que las de Lara (1993), por ejemplo, en el caso del grupo hetero se obtuvo 0.88 y en el grupo homo 0.63 en la escala de masculinidad en comparación con el IMAFE 0.87 . En el caso de la escala de feminidad en el grupo hetero el resultado fue 1.04 y en el grupo homo la media se ubicó en 1.01 comparado con el resultado del IMAFE 1.14. Estos resultados pueden deberse al número de sujetos empleados para cada estudio; Lara (1993) utilizó un grupo de 38 sujetos para el rango de edad analizado (en esta investigación 56). Probablemente, algunos de los factores que pudieron incidir fue el nivel de escolaridad -medio superior y superior- nivel socioeconómico y la edad.

Aunque no es posible asegurar que los sujetos homosexuales sean menos masculinos y más femeninos que los heterosexuales, los resultados muestran que, efectivamente hay un mayor grado de feminidad en el grupo homosexual en comparación con el IMAFE y el grupo heterosexual. Por lo anterior, en principio, es posible aceptar que la orientación sexual determina variaciones en la masculinidad y feminidad de la persona. No obstante, se observó una tendencia a la androginia en el grupo homosexual.

Quizá esto se deba a que los hombres homosexuales se hallan en contextos donde suele predominar gente del mismo sexo, o como señala Garrabe (1993), si la definición de homosexualidad se designa al atractivo erótico por personas del mismo sexo, la preferencia es relativamente continua e incide en el estilo de vida de la persona que la ejerce, sin que ello represente una anomalía mental.

Estadísticamente, no es posible decir que la orientación sexual determina variaciones en la masculinidad y feminidad de los sujetos; ni que las personas homosexuales sean menos masculinas ni más femeninas que las heterosexuales.

\section{Conclusiones}

La homosexualidad no es una patología sino una forma de expresión de la sexualidad del individuo, la cual se encuentra en un ámbito continuo y dinámico; esto es, durante las diferentes etapas de la vida, la persona puede ejercer una orientación sexual hetero, bi u homosexual. Al mismo

12 Jorge García-Villanueva, Denize Maday Meza-Mercado, Claudia Ivonne Hernández-Ramírez y Daniel Moreno-García

Los artículos de la Revista Electrónica Educare del Centro de Investigación y Docencia en Educación de la Universidad Nacional, Costa Rica, se comparten bajo términos de la Licencia Creative Commons: Reconocimiento, No Comercial, Sin Obra Derivada 3.0 Costa Rica. Las autorizaciones adicionales a las aquí delimitadas se pueden obtener en el correo: educare@una.cr 
tiempo, es la constancia de este ejercicio la que determinará muchas características de la sexualidad. En otras palabras, la orientación sexual configura la sexualidad y, a la vez, influye en la expresión de la orientación sexual, de ahí que los estudios acerca del origen de la homosexualidad no tengan términos definitivos, pues desde las diferentes posturas psicológicas se les haya una génesis diversa.

Es un equívoco considerar solamente que la sexualidad puede manifestarse en dos categorías esencialistas -mujer u hombre-, en realidad cada persona es dinámica y puede ejercer las características de los distintos roles sexuales, no solo por su orientación sexual sino por otros motivos y circunstancias que aparecen en cualquier momento de la vida. Las conductas correspondientes a los diferentes géneros son variables en las distintas etapas de la vida y de acuerdo con el tipo de oportunidades sociales que tenga la persona. Esto coincide con lo dicho por Bem (1981), quien señala que un individuo puede ejercer tanto conductas típicamente masculinas como femeninas.

Esta investigación podría plantearse como una transformación radical en la sociedad desde la generación de propuestas que ayuden a la deconstrucción antiesencialista de la diversidad humana y sus múltiples identidades existentes que promuevan la transformación de la ancestral dicotómica entre el sexo y género, propulsando una convivencia diferente entre las personas donde prime el respeto y la tolerancia con la finalidad de poder comprender que el término "sexo es también una construcción social (un efecto repetitivo de performances ritualizadas) y que, por tanto, ha sido género todo el tiempo" (Duque, 2010, p. 33).

\section{Referencias}

Álvarez, J. L. y Mazin, R. (1990). Elementos de sexología. México: McGraw Hill.

Bastin, G. (1979). Diccionario de psicología sexual. Barcelona: Herder.

Bem, S. L. (1981). Gender schema theory: A cognitive account of sex typing. Psychological Review, 88(4), 354-364. doi: https://doi.org/10.1037/0033-295X.88.4.354

Butler, J. (2002). Cuerpos que importan. Sobre los límites materiales y discursivos del sexo. Buenos Aires: Paidós.

Butler, J., Laclau, E. y Žižek, S. (2003). Contigencia, hegemonía, universalidad: Diálogos contemporáneos en la izquierda. Buenos Aires: Fondo de Cultura Económica.

De Barbieri, T. (1993). Sobre la categoría de género. Una introducción teórico-metodológica. Debates en Sociología, 18, 145-169. Recuperado de http://revistas.pucp.edu.pe/index. php/debatesensociologia/article/view/6680/6784

Duque, C. A. (2010). Judith Butler: Performatividad de género y política democrática radical. La manzana de la discordia, 5(1), 27-34. Recuperado de http://bibliotecadigital.univalle.edu. co/bitstream/10893/2675/1/Judith.pdf 
doi: http://dx.doi.org/10.15359/ree.21-2.16

URL: http://www.una.ac.cr/educare

CORREO: educare@una.cr

Fernández, J. (1998). Género y sociedad. España: Pirámide.

Garrabe, J. (1993). Diccionario taxonómico de psiquiatría. México: Fondo de Cultura Económica.

Giménez, R. D. (1992). La homosexualidad. Revista Psicología Práctica, 8.

González, R. (2001). Después de la liberación. Formas transpolíticas, figuras transexuales. México: Universidad Pedagógica Nacional. Recuperado de http://campus.ajusco.upn. mx:8080/upn/bitstream/handle/11195/289/Roberto\%20Gonz\%c3\%a1lez\%20Villareal. pdf? sequence $=1$

Heilman, M. (2012). Gender stereotypes and workplace bias. Research in Organizational Behavior, 32, 113-135. doi: https://doi.org/10.1016/j.riob.2012.11.003

Hernández, R., Fernández-Collado, C. y Baptista, P. (2000). Metodología de la investigación. México: McGraw-Hill.

Historical Notes: A letter from Freud. (1951). The American Journal of Psychiatry, 107(10), 786-787. doi: https://doi.org/10.1176/ajp.107.10.786

Lara, M. A. (1993). Inventario de masculinidad y feminidad IMAFE. México: El Manual Moderno.

Lippa, R. A. (2010). Gender differences in personality and interests: When, where, and why? Social and Personality Psychology Compass, 4(11), 1098-1110. doi: https://doi.org/10.1111/ j.1751-9004.2010.00320.x

Martin, C. L., Ruble, D. N. y Szkrybalo, J. (2002). Cognitive theories of early gender development. Psychological Bulletin, 128(6), 903-933. Doi: https://doi.org/10.1037/0033-2909.128.6.903

McCary, J. L., McCary, S. P., Álvarez-Gayou, J. L., Del Río, C. y Suáez, J. L. (1996). Sexualidad humana. México: El Manual Moderno.

Olvera, G. R. (1997). Perspectivas actuales de la producción teórica sobre masculinidad (Tesis de licenciatura). Universidad Nacional Autónoma de México, México.

Rubio, A. E. y Aldana A. (1994). La expresión homosexual del erotismo. En Antología de la sexualidad humana (Tomo I). México: Consejo Nacional de Población-Miguel Ángel Porrúa.

Valentova, J., Rieger, G., Havlicek, J., Linsenmeier, J. A. y Bailey, J. M. (2011). Judgments of sexual orientation and masculinity-femininity based on thin slices of behavior: A cross-cultural comparison. Archives of Sexual Behavior, 40(6), 1145-1152. doi: https://doi.org/10.1007/ $\underline{\text { s10508-011-9818-1 }}$

Von Krafft-Ebing. (2012). Psychopathia Sexualis. New York: Arcade Publishing. 
doi: http://dx.doi.org/10.15359/ree.21-2.16

URL: http://www.una.ac.cr/educare

CORREO: educare@una.cr

\section{Apéndice A}

\section{INTRUCCIONES}

A continuación encontrará una lista de palabras que describen formas de ser de las personas, por ejemplo: racional, cariñoso, flojo. Le voy a pedir que utilice esas palabras para describirse. Esto es, a cada palabra le pondrá un número entre uno y siete, según qué tan bien crea que describe su manera de ser.

\section{Estos números del uno al siete significan lo siguiente:}

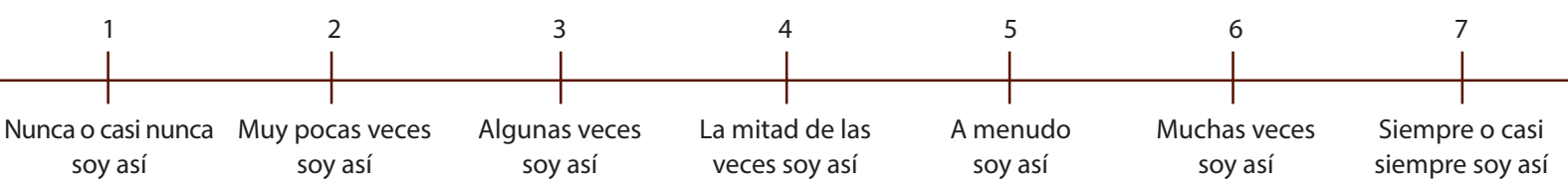

Ejemplo:

Listo $\quad 3$ Le pondrá el número 3 si cree que algunas veces usted es listo.

Malicioso 1 Le pondrá el número 1 si cree que nunca o casi nunca usted es malicioso.

Responsable 7 Le pondrá el número 7 si cree que siempre o casi siempre usted es responsable.

En seguida se encuentran estas descripciones, asigne un número de acuerdo con la escala del 1 al 7 como se muestra arriba.

POR FAVOR NO DEJE NINGÚN INCISO SIN CONTESTAR

\begin{tabular}{|c|c|c|}
\hline 1. Seguro de mí mismo(a) & 21. Dispuesto(a) a arriesgarme & 41. Racional \\
\hline 2. Afectuoso(a) & 22. Deseoso(a) de consolar al que se siente lastimado & 42. Me gustan los niños(as) \\
\hline 3. Enérgico(a) & 23. Agresivo(a) & 43. Rudo(a) \\
\hline 4. Conformista & 24. De personalidad débil & 44. Dependiente \\
\hline 5. Me comporto confiado de los demás & 25. Autosuficiente & 45. Maduro(a) \\
\hline 6. Compasivo(a) & 26. Cariñoso(a) & 46. De voz suave \\
\hline 7. Dominante & 27. Uso malas palabras & 47. Incomprensivo(a) \\
\hline 8. Simplista & 28. Inseguro(a) de mí mismo(a) & 48. Influenciable \\
\hline 9. Analítico(a) & 29. Independiente & 49. Valiente \\
\hline 10. Sensible a las necesidades de los demás & 30. Amigable & 50. Generoso(a) \\
\hline 11. Individualista & 31. Materialista & 51. Frío(a) \\
\hline 12. Sumiso(a) & 32. Pasivo(a) & 52. No me gusta arriesgarme \\
\hline 13. Hábil para dirigir & 33. Competitivo(a) & 53. Reflexivo(a) \\
\hline 14. Comprensivo(a) & 34. Tierno(a) & 54. Espiritual \\
\hline 15. Ambicioso(a) & 35. Autoritario(a) & 55. De voz fuerte \\
\hline 16. Incapaz de planear & 36. Resignado(a) & 56. Retraído(a) \\
\hline 17. Tomo decisiones con facilidad & 37. Atlético(a) & 57. De personalidad fuerte \\
\hline 18. Caritativo(a) & 38. Dulce & 58. Cooperador \\
\hline 19. Arrogante & 39. Egoísta & 59. Malo(a) \\
\hline 20. Indeciso(a) & 40. Cobarde & 60. Tímido(a) \\
\hline
\end{tabular}

